\title{
Neonatal alloimmune thrombocytopenia: two different neonatal manifestations of the same disease
}

\author{
Miguel P. Pereira ${ }^{1 *}$, Marta Pinto², Mafalda Lucas ${ }^{1}$, Ana P. Domingues ${ }^{3}$, \\ Cristina Matos ${ }^{1}$, Jorge Lima ${ }^{3,4}$
}

\begin{abstract}
${ }^{1}$ Department of Pediatrics and Neonatology, ${ }^{3}$ Department of Obstetrics and Gynecology, CUF Descobertas Hospital, Lisbon, Portugal

${ }^{2}$ Department of Obstetrics and Gynecology, CHUC, Coimbra, Portugal

${ }^{4}$ Comprehensive Health Research Centre, CEDOC, NOVA Medical School, Universidade Nova de Lisboa, Lisbon, Portugal
\end{abstract}

Received: 15 May 2021

Accepted: 11 June 2021

*Correspondence:

Dr. Miguel P. Pereira,

E-mail: miguelpereira2112@gmail.com

Copyright: () the author(s), publisher and licensee Medip Academy. This is an open-access article distributed under the terms of the Creative Commons Attribution Non-Commercial License, which permits unrestricted non-commercial use, distribution, and reproduction in any medium, provided the original work is properly cited.

\section{ABSTRACT}

Fetal and neonatal alloimmune thrombocytopenia is a rare disorder in which maternal alloantibodies cross the placenta and cause fetal thrombocytopenia. Most cases are mild but can be potentially fatal if there is an intracranial haemorrhage. Usually, the mother is asymptomatic during pregnancy and no screening is routinely recommended unless there is obstetric or family history. After birth, prompt identification is crucial so that the newborn is closely monitored and treatment is given, if needed, to prevent serious complications. In this article we present two clinical cases of fetal and neonatal alloimmune thrombocytopenia in first-born children, after uneventful pregnancies, with different outcomes and discuss the obstetric management and follow-up in future pregnancies.

Keywords: Neonatal alloimmune thrombocytopenia, Neonatal stroke, Prenatal screening, Antenatal management

\section{INTRODUCTION}

Fetal and neonatal alloimmune thrombocytopenia (FNAIT) is a disorder in which maternal-fetal platelet incompatibility (against fetal platelet antigens inherited from the father, which are absent on maternal platelets) leads to formation of maternal antibodies (IgG) that result in fetal and neonatal thrombocytopenia. Those alloantibodies to human platelet antigen (HPA) cross the placenta and cause fetal platelet destruction and, potentially, suppression of megakaryopoiesis. ${ }^{1}$ The most common antibodies against these antigens, anti- HPA, are anti-HPA-1a (75-80\%) and anti-HPA-5b (10-15\%) but many other were identified. Maternal alloimmunization involves exposure to placental syncytiotrophoblast cells early in pregnancy which explains why FNAIT often affects the first pregnancy of an at-risk couple.
The incidence reported is between 1-1000/10000 births and, in general, the clinical manifestations and the severity of the thrombocytopenia are usually milder in the subsequent pregnancies although the risk of recurrence is 75 to 90 percent. ${ }^{2,3}$ The mother is asymptomatic but the spectrum of fetal/neonatal disease ranges from mild thrombocytopenia to severe intracranial haemorrhage (ICH), in approximately 25 percent of cases, which can be fatal. This disorder requires prompt identification and treatment, and subsequent pregnancies need close surveillance and management. ${ }^{4}$

We present two clinical cases with different initial clinical presentations, highlighting that the most severe cases can have milder presenting symptoms. Both cases have distinct management and outcomes, exhibiting the various spectrums of this disorder. 


\section{CASE REPORT}

\section{Case 1}

A 33-year-old mother with 2 previous miscarriages, gave birth to her first child by vacuum extraction after an uneventful pregnancy (with normal platelet and leucocyte counts). She had no history of medications, blood transfusions or hepatitis B infection. The male infant's birth weight was $2990 \mathrm{~g}$ with Apgar scores of 9,9 and 10 at 1,5 and 10 minutes, respectively. Immediately after birth, the newborn was noted to be irritable and physical examination revealed generalized petechiae and skin pallor. The newborn was admitted to the neonatology intensive care unit (NICU).

The newborn's platelet count was $4 \times 10^{9} / 1$, activated partial thromboplastin time (APTT) 27.8 seconds, international normalized ratio (INR) 2 and a C-reactive protein (CRP) $0.02 \mathrm{mg} / \mathrm{dl}$. Red blood cells (RBC) and white blood cell (WBC) counts were within the normal range. Blood cultures were negative and blood glucose, blood gas analyses and thorax X-ray were normal.

The cranial ultrasound showed a parenchymatous hyperechoic lesion, of triangular shape, on the left frontoparietal region, close to the ventricle. A cranioencephalic magnetic resonance was performed revealing the presence of blood in the frontoparietal intra axial space of the middle left cerebral convexity, findings compatible with a haemorrhagic stroke (Figure 1 and 2).

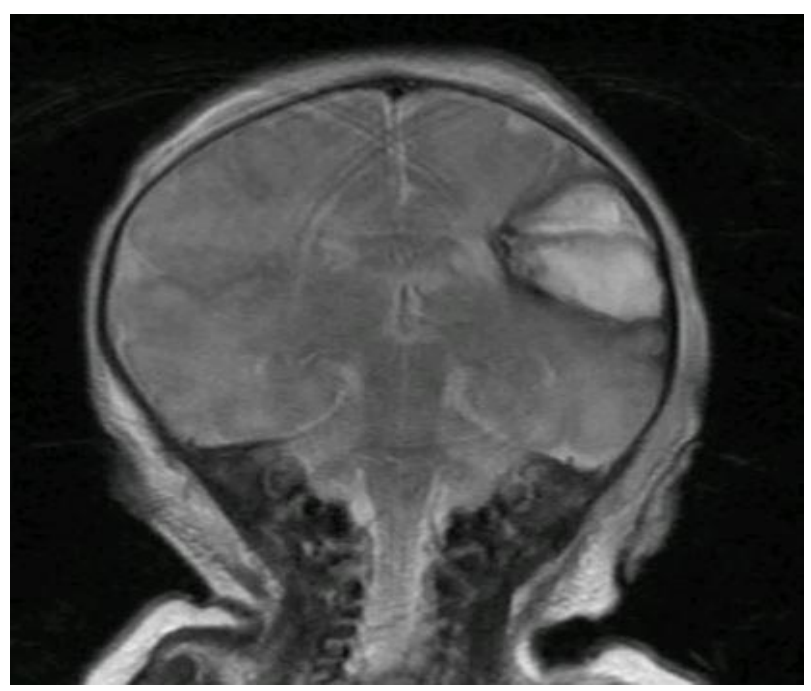

Figure 1: A coronal view of the brain magnetic resonance imaging - case 1 - showing blood in the frontoparietal intra axial space of the middle left cerebral convexity.

Serum samples of the infant and his parents were taken for HPA-antibody testing and genotyping, to determine offspring risk. A maternal-fetal platelet mismatch for HPA-1a was found.

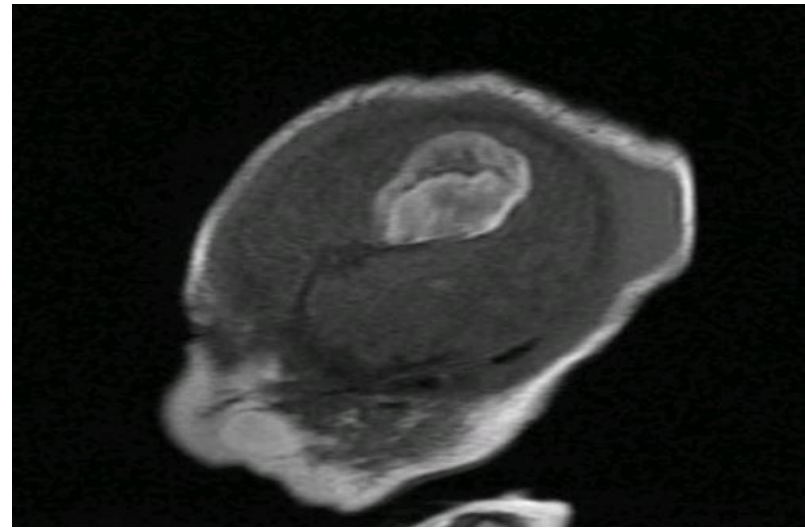

Figure 2: Haemorrhagic stroke in a sagittal view case 1.

A platelet transfusion $(10 \mathrm{ml} / \mathrm{kg})$ was given after birth. Two daily doses of intravenous immunoglobulin $(1 \mathrm{~g} / \mathrm{kg})$ were given in the second and third days of life.

Twelve hours after the first platelet transfusion, a cephalohematoma was observed, that gradually reabsorbed in the first week of life. There was no progression of petechiae after birth. The platelet count was $76 \times 109 / 1$ by the $2^{\text {nd }}$ day of life and $91 \times 109 / 1$ by the $3 r d$. During hospitalization, the newborn went through serial neurologic examinations, being noticed a persistent axial hypotonia, a hypertonia of the lower limbs (more evident on the right), and an asymmetry of the plantar reflex (nonflexor on the right foot). The newborn was on exclusive enteral feeding since the fifth of life, with autonomy since the tenth and was discharged home on the fourteenth day of life. A neuropediatric consultation at a neurodevelopment centre was scheduled.

\section{Case 2}

A 37-year-old woman, nulliparous, gave birth to her first child by an elective caesarean section (C-section) (due to a previous pelvic orthopaedic surgery) after an uneventful pregnancy (with normal platelet and leucocyte counts). She had no history of medications, blood transfusions or hepatitis B infection. The male infant's birth weight was $3250 \mathrm{~g}$ with Apgar scores of 9,10 and 10 at 1,5 and 10 minutes, respectively. At birth, multiple petechiae and haemorrhagic suffusions were observed and the newborn was admitted to the NICU (Figure 3).

The newborn's platelet count was $6 \times 10^{9} / \mathrm{l}$, APTT 34.4 seconds, INR 1.53 , and a CRP $0.11 \mathrm{mg} / \mathrm{dl}$. RBC and WBC counts were within the normal range and blood cultures were negative.

During the follow-up, the newborn was submitted to several head ultrasounds, all of them were normal.

Serum samples of the infant and his parents were also tested and a maternal-fetal platelet incompatibility for both HPA-1a and HPA 15a was revealed. 
The newborn had the first platelet transfusion $(15 \mathrm{ml} / \mathrm{kg})$ after birth. A total of 9 platelet transfusions were given during hospitalisation. There were also administered three daily doses of intravenous immunoglobulin $(1 \mathrm{~g} / \mathrm{kg})$. A 5day course of methylprednisolone $(2 \mathrm{ml} / \mathrm{kg} /$ day $)$ was completed between ninth and fourteenth days of life.

By the second day of life, platelet count rose to $58 \times 10^{9} / 1$, however, in the following 2 days, a sharp drop was found, reaching $9 \times 109 / 1$ by the ninth day of life. After the eleventh day of life, platelet count was always higher than $50 \times 109 / 1$. The newborn was discharged home by the fourteenth day after birth with a platelet count $191 \times 10^{9} / 1$ and there was no further progression of petechiae and haemorrhagic suffusions. The newborn has been on exclusive enteral feeding with autonomy since birth and had an unremarkable neurologic examination.

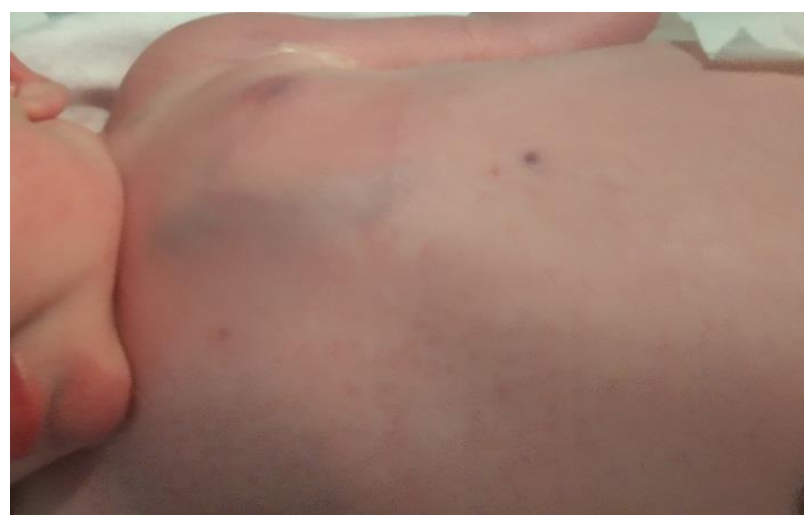

Figure 3: Petechiae and haemorrhagic suffusions case 2.

\section{DISCUSSION}

Neonatal thrombocytopenia is defined as a platelet count $<150 \times 10^{9} / 1$ and it is classified as severe if the value is $<50 \times 10^{9} / 1$. FNAIT has a broad clinical spectrum and clinical findings are dependent on the severity of thrombocytopenia. In neonates with moderately severe to severe thrombocytopenia, petechiae, bruising, and bleeding may be observed, but the most serious complication is $\mathrm{ICH}^{5}$ The reported risk of $\mathrm{ICH}$ ranges from 10 to 25 percent of newborns with FNAIT, being higher for infants born to multiparous women, and especially for those who have had an older affected sibling. ${ }^{6}$ One-quarter to one-half of these incidences take place in utero and $54 \%$ occur before 28 weeks gestation. The consequences include death $(35 \%)$ or serious neurological sequelae in up to $83 \%$. $^{7}$

At birth, neonatal platelet counts often are less than $100,000 /$ microlitre. The platelet count typically falls in the first few days after birth, then rises over the next one to four weeks as the antibody level declines. ${ }^{5}$ In the case 1 the newborn platelet count was classified as severe $\left(4 \times 10^{9} / 1\right)$ and the newborn presented signs of irritability due to ICH.
In case 2 the clinical signs of petechiae and haemorrhagic suffusions were due to a platelet count of $6 \times 10^{9} / 1$.

As described in the presented cases, the diagnosis of FNAIT should be considered in any otherwise wellappearing term infant who presents with thrombocytopenia at birth or within seven days after birth, but most cases are diagnosed when a child is born with petechiae or other signs of bleeding in the absence of any other condition.

Autoimmune thrombocytopenia can be confused with FNAIT. In FNAIT, the mother has a normal platelet count and only the fetal platelets are affected while in autoimmune thrombocytopenia the mother has autoantibodies which affect both maternal and fetal platelets. FNAIT is more severe for the fetus and neonate and, on the other hand, the clinical manifestations of autoimmune thrombocytopenia are typically milder.

Serologic testing demonstrates the presence of maternal HPA antibodies in the infant..$^{5}$ Although there are several commercially available laboratory assays, the monoclonal antibody immobilization of platelet antigen (MAIPA) assay is the gold standard but given that it usually takes 3 days, which is too long for neonates to wait for treatment, they are best used to confirm the diagnosis and to monitor the maternal antibody level in the subsequent pregnancies. ${ }^{8}$

A cranial ultrasound should be performed in any affected infant with a platelet count $<50,000 /$ microlitre because of the high rate of intracranial haemorrhage. It should be obtained as soon as possible after delivery to determine the severity and management. ${ }^{5}$

During the first days of life, the platelet count should be closely monitored. Once a trend showing an increase in platelet count is observed, the interval between testing is increased until the count is stable. Term infants who are not ill and have no other risk factors for haemorrhage should be transfused if the platelet count is $<30 \times 10^{9} / 1$ or if there are signs of major bleeding, defined as $\mathrm{ICH}$, pulmonary haemorrhage, frank rectal bleeding, lifethreatening bleeding requiring emergency fluid therapy, or red blood cell transfusion. The threshold is higher $\left(<50 \times 10^{9} / 1\right)$ in infants who are ill or have additional risk factors (such as a previous sibling with FNAIT or presence of ICH on a postnatal head ultrasound), as described in case 1 of this article in which the newborn received a platelet transfusion after birth. If a platelet transfusion is administered, the platelet count should be maintained between $50 \times 10^{9} / 1$ and $100 \times 10^{9} / 1$ in infants who have evidence of ICH. Adequate platelet counts should be maintained during the first 72 to 96 hours because the risk of intracranial haemorrhage is highest during this period. After that period, the decision to transfuse depends upon clinical circumstances. ${ }^{5}$ 
Many neonates' exhibit signs of bleeding during or immediately after delivery and appropriate therapies are not always readily available. Transfusion of donormatched, antigen-negative platelets is the treatment of choice. ${ }^{9}$ In up to $95 \%$ of cases, platelets from HPA-1a- and HPA-5b-negative individuals are compatible and effectively increase platelet counts with improved survival. ${ }^{8}$ Unfortunately, few hospitals keep genotyped platelets stored. If compatible platelets are not available, and if the mother is suitable for platelet apheresis, it is possible to transfuse the newborn with washed, irradiated and leuko-reduced platelets harvested from the mother before delivery. Since this takes several hours, this approach can only be used in cases where FNAIT is expected. Another treatment option is the immediate transfusion of random donor platelets, being primarily used as an alternative strategy to temporarily increase platelet counts until compatible platelets become available. ${ }^{10,11}$

Treatment with high-dose intravenous immune globulin (IVIg) often is effective in prolonging the survival of transfused platelets. It could be given to patients with FNAIT immediately following platelet transfusion. There is limited evidence that IVIg should be used alone. Intravenous methylprednisolone has been used by some as an adjunctive therapy, however, studies on its efficacy are lacking. It could be considered in instances where lifethreatening thrombocytopenia persists despite platelet transfusions and IVIg. 5,12

The prenatal approach is controversial. Both cases discussed were first-born children and although screening is not routinely recommended the management of following pregnancies is specific. It is important to counsel the parents that the history of a previously affected child, especially if that child had $\mathrm{ICH}$, is the strongest predictor of recurrence and severity of FNAIT in a future pregnancy. ${ }^{13}$

The more commonly detected antibodies in Caucasians are those against HPA-1a in $80 \%$ of cases, which cause a more severe disease. Anti-HPA-5b alloimmunization is found in 10 to $15 \%$ and is less severe. The presence of maternal HLA-DRB3 0101 has a positive predictive value of $35 \%$ for clinically significant FNAIT. ${ }^{14}$

The mother is asymptomatic, and the aim of screening pregnant women would be to detect the condition during the mother's first pregnancy to decrease morbidity and mortality associated with FNAIT. The screening would be only for anti-HPA-1a (which causes $95 \%$ of severe FNAIT) but is costly, the prevalence in the population is low $(0.1$ to $0.2 \%)$ and the optimal management is still unknown. Maternal and paternal platelet antigen genotyping, as well as maternal human anti-platelet antibody evaluation should be performed when the woman or her sister has an obstetric history suggestive of this diagnosis. $^{15}$
In both cases presented, a maternal-fetal platelet incompatibility for HPA-1a was found. The antigen typing of the mother's and father's platelets and the mother's serum should be tested for antiplatelet alloantibodies, although the antibody titter does not correlate well with severity. ${ }^{5}$

In all cases, whether the father is homozygous (nearly $100 \%$ recurrence rate in subsequent pregnancies) or heterozygous for the causative HPA, fetal HPA genotyping should be performed by chorionic villus sampling or amniocentesis (fetal genotyping using a cellfree fetal DNA test is not commercially available). ${ }^{8}$

The antenatal management of FNAIT in subsequent pregnancies remains controversial. The treatment involves three options: maternal intravenous immunoglobulin (IVIg), maternal steroid administration, or serial intrauterine platelet transfusions (IUT). Both maternal IVIg and IUT can prevent severe fetal thrombocytopenia and its complications, but IUT is associated with increased fetal haemorrhage and intrauterine death. If so, it is preferable a conservative approach and close monitoring. ${ }^{16}$

Women should initiate therapy with IVIg between 12 and 20 weeks of gestation (WOG), depending on the severity of FNAIT. The maternal treatment with IVIg produces a significant increase in the platelet count of fetuses and is the first-line therapy because of the risks of fetal loss due to weekly platelet intrauterine transfusions (IUT) and fetal blood samples (FBS) by umbilical blood sampling as done in the past. However, some authors are concern that omitting the FBS (usually done around 6 to 8 weeks after starting treatment) disables the opportunity to identify non-responders and in that case, increasing the dose of IVIg, adding steroids or weekly platelet IUT would improve the outcome. Nevertheless, the risks of FBS outweigh the benefits and is not routinely recommended but may have a place for high-risk women. ${ }^{17}$

Ultrasound examinations should be performed at four-to six-week intervals beginning at 16 to 20 weeks and continuing until delivery since ICH can occur antenatally in pregnancies at risk of FNAIT. ${ }^{15}$

The timing for delivery lays on a delicate balance between removing the fetus from the source of anti-platelet antibodies and minimizing the risks of preterm birth. Elective caesarean section at 37 weeks may help prevent ICH from occurring, so it is the preferred mode of birth with a course of steroids prior to delivery (indicated for lung maturity rather than to increase fetal platelet count). In extremely high-risk pregnancies, C-section may be considered at 36 weeks. ${ }^{18}$

Vaginal delivery is considered a reasonable alternative for women in the standard and high-risk categories, only if percutaneous umbilical blood sampling at or after 32 weeks shows more than $100 \times 10^{9} / 1$ platelets. Induction of labour at 38 weeks with avoidance of rotational or vacuum 
delivery or fetal blood sampling (FBS) in labour is recommended if the woman is multiparous. ${ }^{18}$

\section{CONCLUSION}

FNAIT is an uncommon disease, and it can cause severe fetal and neonatal complications, especially if $\mathrm{ICH}$ develops, with long-term consequences. The diagnosis should be suspected if a neonate presents with unexplained haemorrhage or if there is a family history of FNAIT.

As described in the clinical cases, neonates should be closely monitored and the platelet count should be kept above $30 \times 10^{9} / 1$, if no other risk factors. A cranial ultrasound should be made to discard ICH.

At this time, there is no cost-effective validated screening test for this pathology, so universal screening is not recommended. Regardless, diagnosing FNAIT is important in subsequent pregnancies, to avoid recurrences. Treatment with maternal intravenous immunoglobulin (first-line therapy) and a regular ultrasound monitoring are the foundations of prenatal management.

\section{Funding: No funding sources}

Conflict of interest: None declared

Ethical approval: Not required

\section{REFERENCES}

1. Liu Z, Bussel JB, Lakkaraja M, Ferrer-Marin F, Ghevaert C, Feldman HA, et al. Suppression of in vitro megakaryopoiesis by maternal sera containing anti-HPA-1a antibodies. Blood. 2015;126(10):12346.

2. Bussel J, Zabusky MR, Berkowitz RL, McFarland JG. Fetal alloimmune thrombocytopenia. N Engl J Med. 1997;337:22-6.

3. Knight M, Pierce M, Allen D, Kurinczuk JJ, Spark P, Roberts DJ, et al. The incidence and outcomes of fetomaternal alloimmune thrombocytopenia: a UK national study using three data sources. $\mathrm{Br} \mathrm{J}$ Haematol. 2011;152(4):460-8.

4. Kamphuis M, Paridaans NP, Porcelijn L, Lopriore E, Oepkes D. Incidence and consequences of neonatal alloimmune thrombocytopenia: a systematic review. Pediatrics. 2014;133(4):715-21.

5. Caraciolo JF. Neonatal immune-mediated thrombocytopenia. Available at: https://www.upto date.com. Accessed on 05 May 2020.

6. Delbos F, Bertrand G, Croisille L, Ansart-Pirenne H, Bierling P, Kaplan C. Fetal and neonatal alloimmune thrombocytopenia: predictive factors of intracranial hemorrhage. Transfusion. 2016;56(1):59-66.

7. Tiller H, Kamphuis MM, Flodmark O, Papadogiannakis N, David AL, Sainio S, et al. Fetal intracranial haemorrhages caused by fetal and neonatal alloimmune thrombocytopenia: an observational cohort study of 43 cases from an international multicentre registry. BMJ Open. 2013;3(3):e002490.

8. Zdravic D, Yougbare I, Vadasz B, Li C, Marshall AH, Pingguo Chen, et al. Fetal and neonatal alloimmune thrombocytopenia. Sem Fetal Neonat Med. 2016:21(1):19-27.

9. Lieberman L, Greinacher A, Murphy MF, Bussel J, Bakchoul T, Corke $\mathrm{S}$ et al. Fetal and neonatal alloimmune thrombocytopenia: recommendations for evidence-based practice, an international approach. $\mathrm{Br}$ J Haematol. 2019;185(3):549-62.

10. Allen D, Verjee S, Rees S, Murphy MF, Roberts DJ. Platelet transfusion in neonatal alloimmune thrombocytopenia. Blood. 2007;109(1):388-9.

11. Bakchoul T, Sachs UJ, Wittekindt B, Sclösser R, Bein G, Santoso S. Treatment of fetomaternal neonatal alloimmune thrombocytopenia with random platelets. Pediatr Blood Cancer. 2008;50:1293-4.

12. Peterson J, McFarland JG, Curtis BR, Aster RH. Neonatal alloimmune thrombocytopenia: pathogenesis, diagnosis and management. $\mathrm{Br} \mathrm{J}$ Haematol. 2013;161(1):3-14.

13. Bussel J, Berkowitz RL, Hung C, Kolb EA, Wissert $\mathrm{M}$, Primiani A, et al. Intracranial hemorrhage in alloimmune thrombocytopenia: stratified management to prevent recurrence in the subsequent affected fetus. Am J Obstet Gynecol. 2010;203(2):135.

14. Killie MK, Husebekk A, Kjeldsen-Kragh J, Skogen B. A prospective study of maternal anti-HPA 1a antibody level as a potential predictor of alloimmune thrombocytopenia in the newborn. Haematologica. 2008;93(6):870-7.

15. Paidas MJ. UpToDate. Fetal and neonatal alloimmune thrombocytopenia: Parental evaluation and pregnancy management. Available at: https://www.uptodate.com. Accessed on 05 May 2020.

16. Pacheco LD, Berkowitz RL, Moise KJ Jr, Bussel JB, McFarland JG, Saade GR. Fetal and neonatal alloimmune thrombocytopenia: a management algorithm based on risk stratification. Obstet Gynecol. 2011;118(5):1157-63.

17. Rayment R, Brunskill SJ, Soothill PW, Roberts DJ, Bussel JB, Murphy MF. Antenatal interventions for fetomaternal alloimmune thrombocytopenia. Cochrane Database Syst Rev. 2011;(5):CD004226.

18. Regan F, Lees CC, Jones B, Nicolaides KH, Wimalasundera RC, Mijovic A, et al. Prenatal Management of Pregnancies at Risk of Fetal Neonatal Alloimmune Thrombocytopenia (FNAIT). BJOG. 2019;126(10):173-85.

Cite this article as: Pereira MP, Pinto M, Lucas M, Domingues AP, Matos C, Lima J. Neonatal alloimmune thrombocytopenia: two different neonatal manifestations of the same disease. Int $\mathbf{J}$ Reprod Contracept Obstet Gynecol 2021;10:2867-71. 